\title{
MATHEMATICAL MODELING OF CONTACT THERMOCOUPLE
}

\author{
T. V. Borovkova, V. N. Yeliseyev \\ Moscow State Technical University, Moscow \\ I. I. Lopukhov \\ NPO Mashinostroeniya, Reutov, Russia
}

The contact thermocouples introduce method errors into the thermal tests results. The influence of the parameters, like the density of the incident thermal flux, the inaccuracy of the thermocouple position in the investigated construction element groove, thermal characteristics of the groove materials, on the method error is given. The obtained results allow one to increase the precision of temperature measurement at the construction unit thermal tests.

Контактные термопары вносят ошибку метода в результаты тепловых тестов. Рассмотрено влияние на ошибку метода параметров, таких как плотность падающего теплового потока, неточность положения термопары в пазе исследуемого элемента конструкции, термальные характеристики материала паза. Полученные результаты позволяют повысить точность температурных измерений в элементах конструкции при тепловых тестах.

PACS: 02.60.Cb; 07.20.Dt

Thermocouple sensors are widely used at the thermal tests of the aircrafts construction units on the radiation heat test bench for the thermal regime reproduction control. Such measurements with the help of the thermocouple sensors of the instrumental errors, caused by nonlinear thermal properties, and structure changes etc., with the help of thermocouple sensors are given in articles [1,2]. Different analytical solutions of the stationary task about the method errors of construction surface temperature measurement are produced in the article [3]. Also there are extensive and detailed studies of the method errors that appeared at the setting of the welded and glued thermocouple sensors for different heated conditions in the thin-walled metal construction [4]. Moreover, ceramic, composition, and laminated materials permitted of the various contact sensors setting ways are widely used at up-to-date constructions designing. The information about method errors of temperature measurements in such materials is unsystematized and fragmentary.

The investigation results of the temperature field measurement errors of the composite sample at the setting of the sensor into the groove at some depth are presented in this article. The thermal conductivity value of the composition materials that are used in the up-to-date aircraft at high thermal load is one or two orders less than the same index for the metals. It leads to the appearance of the large temperature gradient dependent on the sample thickness. In practice the reproduction of the dynamical temperature field on the sample surface at the thermal testing is often realized by thermocouples that are placed at some depth near the 
heated surface without taking the method errors into account. In this case the method errors are determined by the dramatically difference between the temperature on the surface and the one in the sensor setting place.

One can assert that taking into account the high accuracy of the modern measuring instruments, the decrease of the measurements precision is the result of the method error influence generally [4]. On numerous occasions two kinds of method errors like thermocouple errors measuring the temperature near the contact area, and errors caused by the heat abstraction through thermocouple thermoelectrodes make the most essential effect on the temperature measurement precision [3]. In many cases it is so difficult to estimate these errors as they depend on a great number of the factors. Nevertheless we succeeded to determine the error value for a whole series of tasks owing to the creation of the $3 \mathrm{D}$ model of the sample used for heat runs (Fig. 1).

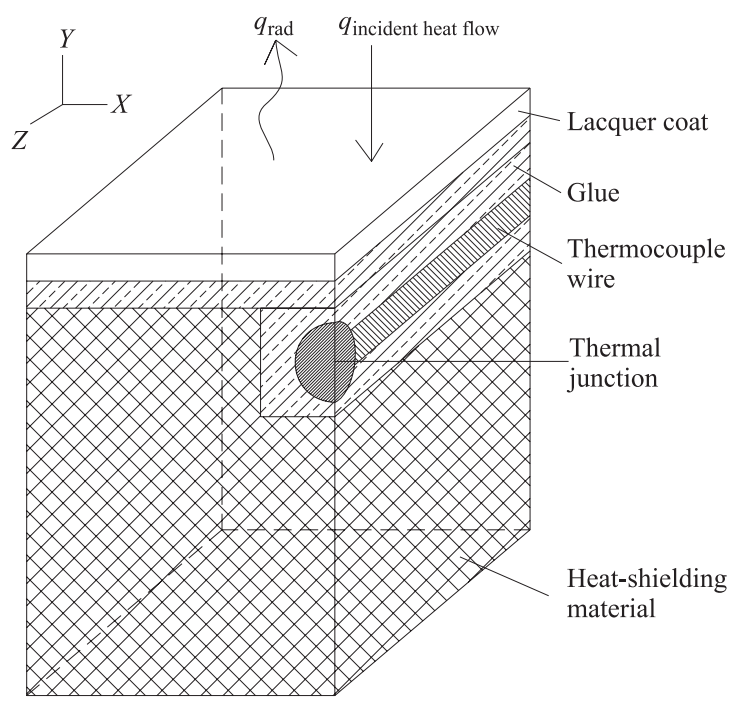

Fig. 1. The model of the unit under test

The sample is a parallelepiped of the heat-shielding material with the rectangular groove $0.6 \times 0.6 \mathrm{~mm}$, and the length is $6 \mathrm{~mm}$. The chromel-alumel thermocouple is placed into it (the thermoelectrodes diameter is $0.2 \mathrm{~mm}$, the thermojunction diameter is $0.5 \mathrm{~mm}$ ). The thermocouple model is an aggregate of the separate construction units; and the thermoelectrodes are the cylindrical rods possessing an ideal contact with the ball that is simulated the thermojunction. The propagation of the heat through the thermoelectrodes is taken into account in the analysis. With the purpose of the fixation of the thermocouple the groove is filled with the glue. The whole heating surface is covered by the layer of the lacquer coating to make it equally black $(\epsilon=0.9)$. Unless another is said, let us consider that the thermocouple axis coincides with the longitudinal symmetry axis of the groove.

In Fig. 2 the layout of the control points is represented: $T_{1}$ is the temperature of the heated surface of the unit under test far from the thermocouple setting place, $T_{2}$ is the temperature in the center of the thermojunction if the longitudinal thermocouple axis coincides with the longitudinal symmetry axis of the groove, $T_{3}$ is the temperature in the point placed far from 
the thermocouple setting place at the same depth as the sensor junction center, $T_{4}$ is the temperature of the heated surface of the unit under test in the point placed on the vertical axis passing through the sensor junction center (the distance between point couple 4 and 2,1 and 3 through the axis is equal to $0.56 \mathrm{~mm}), T_{5}$ is the temperature in the junction center if the longitudinal axis is removed upstairs relative to the groove axis for a distance of $0.005 \mathrm{~mm}$. The choice of the control points place is connected with the various exploration problems.
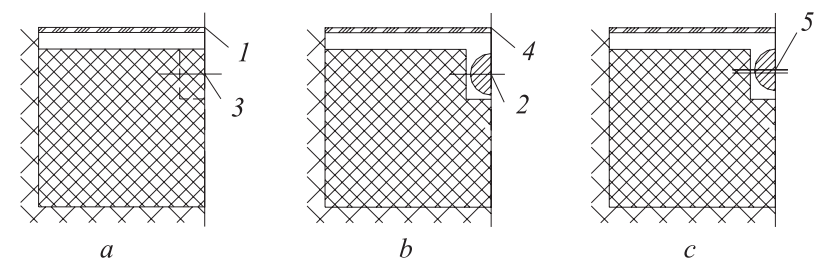

Fig. 2. Control points: $1-T_{1} ; 2-T_{2} ; 3-T_{3} ; 4-T_{4} ; 5-T_{5}$

For example, if it is needed to determine the true temperature in the thermojunction setting place, it is expedient to compare the temperature in points 3 and 2 . If the thermocouple junction is placed at some depth, and if it is necessary to control the temperature of the sample surface under the experiment with the help of such thermocouple registrations, it is convenient to compare the temperature in points 1 and 2 , etc.

The solution of the 3D nonstationary thermal conductivity problem is found with the help of the finite-element method realized by the program package Nastran. The finite elements (FE) in the form of the tetrahedron are used in the simulation of the sample-sensor system. This form of the FE is stipulated the rather precise and the higher stable simulation of the thermoelectrodes and the thermojunction compared with the case when the FE have the parallelepiped form. The sample is heating by the radiant heat flow with the constant value that changes from 50 to $250 \mathrm{~kW} / \mathrm{m}^{2}$. In that way the resulting flow is the difference of the absorbed and irradiated flows (Fig. 3):

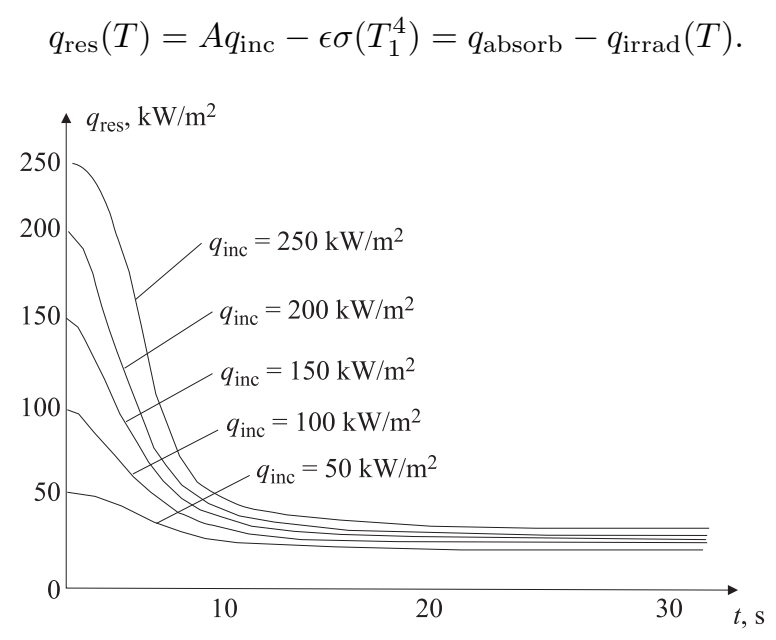

Fig. 3. The resulting flow 
It is clear that the most marked change of the $q_{\mathrm{res}}$ occurs in the interval from 0 to $5 \mathrm{~s}$, and depends on the incident flow value. By the simulation on the lateral and lower faces of the sample-sensor system the thermal flow takes on a zero value.

The geometrical model is a quarter of the real unit under test in the investigation. The possibility of such a simplification is governed by the symmetry of the sample and the fact that the properties difference of two thermoelectrodes materials has a marginal effect on the method errors. In [5] it was shown that the values $\triangle T_{12}(t)$ differ less than $0.15 \%$ for chromel and alumel thermoelectrodes for the same value of the thermal flow density. That is why it is acceptable to consider the thermocouple with the thermoelectrodes having the same properties for the calculations time reduction.

Also the thermocouple forms often used in the calculations of the thermal field by the analytical methods of the simplification are investigated. The sensor may be presented as the rode with the square section having the same surface square as the rode with the circular section. The results obtained with the help of models $4 \mathrm{a}$ and $4 \mathrm{~b}$ were compared with the results of the model of the thermocouple with the spherical junction (see Fig. 1).

The using of the simplified sensor models in such calculation methods as the method of elementary balances, analytical methods, etc., is warrantable because the method errors values introduced by each of the considered thermocouple model differ one from each other not more than to $1 \%$ by the mentioned thermal influence.

The heating by the thermal flow creates on the surface of the unit under test the definite temperature regime $T_{1}(t)$. However, in practice at the thermal tests the registrations of the thermocouple sensor placed at the definite depth into the specimen material $T_{2}(t)$ are used for the dynamical temperature surface field control.

The method error in that case is determined by the gradient of the temperatures dependent on the sample depth and the presence of the thermocouple inside the material.

The influence of the temperature gradient on the method error is governed by the difference of the temperatures $\triangle T_{13}(t)=T_{1}-T_{3}$, but the distorting effect of the foreign bodies (thermocouple and glue) is governed by the difference of the temperatures $\triangle T_{32}(t)=T_{3}-T_{2}$. As a result, the method error value is equal to the difference $\triangle T_{12}(t)=T_{1}-T_{2}=\triangle T_{13}(t)+$ $\triangle T_{32}(t)$ (Fig. 4).

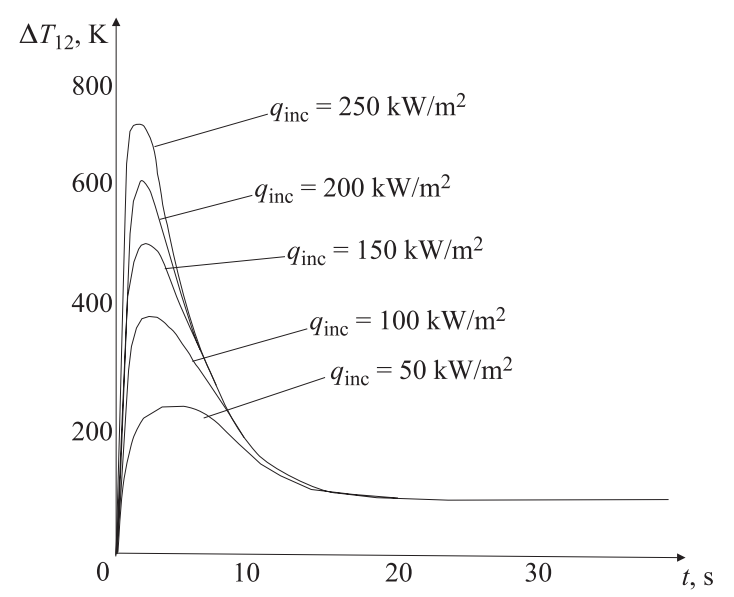

Fig. 4. The dependence of the method error on time for different values of the incident thermal flow 
It is clear from Fig. 4 that the maximum error value is achieved on the first stage of the experiment. The heating character determines it. On the picture the approach of the curves on the time interval from the 15 th second means that the character of the process becomes «regular», and the error value depends heavier on the geometric and thermal characteristics of the sample-sensor system, than on the incident thermal flow density.

The thermocouple may move from the longitudinal groove axis, for example, to the surface (in this case the quantity of the removal is equal to $\Delta h_{t c}=0.05 \mathrm{~mm}$, which is about $8 \%$ of the groove depth). That removal of the thermosensor from the longitudinal groove axis may also influence the method error. In that case the error is determined by the temperature gradient through the sample thickness and takes into account the temperature difference in the junction center of the «unremoved» and «removed upwards» thermocouples: $\triangle T_{15}(t)=T_{1}-T_{5}=\triangle T_{12}(t)+\triangle T_{25}(t)$.

The performed investigation showed that the contribution of the inaccuracy of the thermocouple setting place to the considered thermal flows just on the initial heat stage is not more than $5 \%$ with the inaccuracy of the thermocouple setting place not more than $8 \%$.

During the investigation of the method errors dependence on the thermal properties of the glue it is ascertained that if we change its density and thermal capacity twofold, their influence on the method error is of great importance in the interval till $20 \mathrm{~s}$ and is about $15 \%$. And if we change glue's thermal conductivity twofold, its influence on the method error is of great importance in the whole considered interval and is about $11 \%$. During the investigations it was determined that the influence quantity of the glue's thermal capacity and density on the method error is larger than the influence quantity of the thermal conductivity. It means that the material with the lower value of the product $C \rho$ solid thermal capacity is preferable.

For the changing of the construction thermal field, for example, for its smoothing, the radiation properties of the surface may be used (for instance, $\epsilon$ is the blackness value). In this case during the preparation for the experiment the cover with the variable coefficient $\epsilon$ is placed on the unit under test surface. In that way the cover has the maximum value of the blackness in the area of the surface under the thermocouple. The variation interval of the $\epsilon$ through the heat surface is considered from 0.2 to 0.8 (Fig. 5).

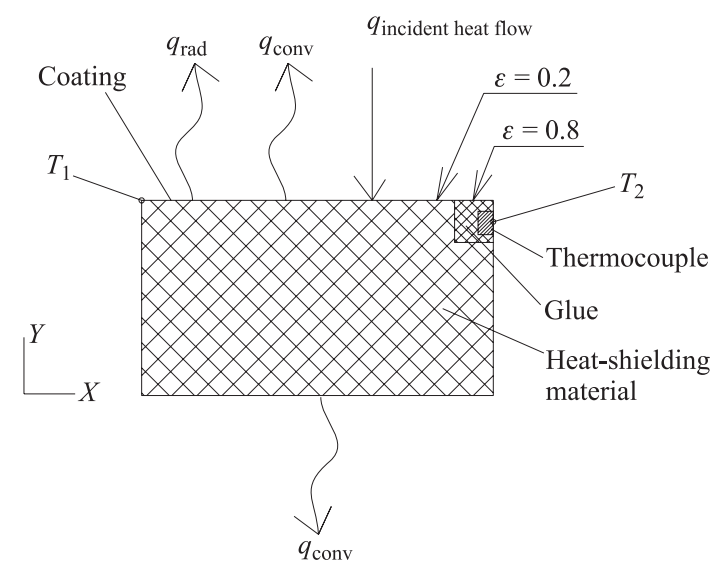

Fig. 5. The model for the investigations of the influence of $\epsilon$ to the method error 
For the investigation of the influence on the method error of the blackness value the calculations of the temperature field of the sample material with the constant value of $\epsilon$ on the surface in the interval from 0.2 to 0.8 is performed.

The value of the method error is determined by the following formula: $\triangle T_{12}(t)=T_{1}-T_{2}$ (Fig. 6).

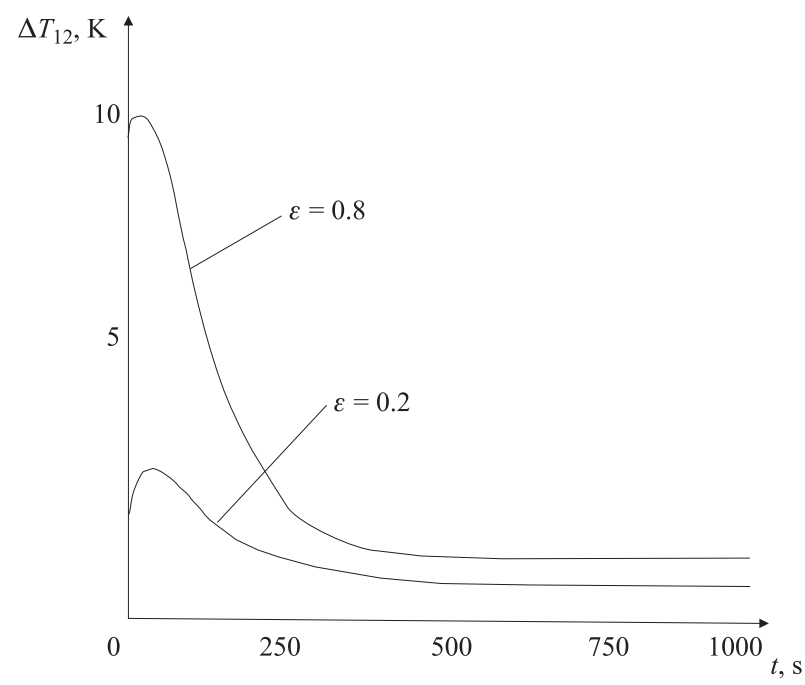

Fig. 6. The dependence of the method errors on the blackness value of the cover

During the investigations it was ascertained that the value of the method error increases with the increase of $\epsilon$. And the method error value achieves its maximum value in the interval from 0 to $100 \mathrm{~s}$. In the interval below $600 \mathrm{~s}$ the increase velocities of temperatures $T_{2}(t, \epsilon=0.2)$ and $T_{2}(t, \epsilon=0.8)$ dramatically differ, so the increase velocity of the method error for the cover with the $\epsilon=0.8$ is larger than that for the cover with $\epsilon=0.2$.

To increase the precision of the temperature measurements and the accuracy of the definite temperature regime reproduction of the construction elements from the low heat-conducting materials on the surface during the tests on the radiation testing bench, the following is necessary:

- to obtain the dependence of the temperature $T_{2}(t)$ experimentally;

- to calculate the dependence of $\triangle T_{12}(t)$ taking into account the probable removal displacement of the thermocouple, the properties of the glue, $q_{\text {inc }}$, and the heated surface blackness quantity;

- to use the values of $T_{1}$ adjusted taking into account the method error value in the automatic bench operation system: $\triangle T_{12}(t)=T_{2}(t)+T_{12}(t)$.

If the definition of the material thermal properties is the aim of the tests, then the required dependence of the temperature in the point the coordinate of which coincides with the thermosensor junction setting place $T_{3}(t)$ (see Fig. 2) may be obtained by the calculations taking into account the method error value $\triangle T_{32}(t)$ with the help of the following formula: $\triangle T_{3}(t)=T_{2}(t)+T_{32}(t)$. 
472 Borovkova T.V., Yeliseyev V.N., Lopukhov I. I.

\section{REFERENCES}

1. Elyseev V.N. et al. The Error Estimation of the Surface Temperature Measurement of the Semitransparent Material by the Contact Sensor // IVUS. Mashinostroenie. 1981. V.11. P.77-81 (in Russian).

2. Reznik S. V. et al. Identification of Radiative and Conductive Heat Transfer Parameters at Presence of Errors in Initial Data // Inverse Problems and Exp. Design in Thermal and Mech. Engineering: Proc. of Eurotherm. Seminar. 2001. V.68. P. 293-300.

3. Kulakov M.V., Makarov B.I. The Measurement of the Temperature on the Solid States Surface. M.: Energia, 1979.

4. Baranov A. N. et al. The Method Errors of the Measurement by Thermocouples of the Thin-Walled Metal Construction Temperature // Tr. TsAGI. 2002. No. 2658 (in Russian).

5. Borovkova T.V., Elyseev V.N., Lopukhov I.I. The Increase of the Precision of the Temperature Measurement during the Tests of the Construction Elements of Low Heat-Conducting Materials on the Radiation Heated Test Bench // Vestn. MGTU. Mashinostroenie. 2006. V.3. P.51-63 (in Russian). 